\title{
EDITORIAL
}

\section{ENFERMERÍA, SALUD Y CIENCIAS SOCIALES: UN PUENTE NECESARIO}

\author{
NURSING, HEALTHCARE AND THE SOCIAL SCIENCES: \\ A NECESSARY LINK \\ $\mathrm{PHD}^{\odot}$ Ricardo A. Ayala \\ Programa de Doctorado en Sociología, Universidad de Ghent, Bélgica
}

\begin{abstract}
T os puentes interdisciplinarios son hoy en día un campo privilegiado de ex$\_$pansión del conocimiento en los aspectos en que una disciplina por sí sola no puede resolver. El fértil campo de la sociología de la salud, por ejemplo, es un movimiento internacional que ha experimentado un crecimiento sostenido, con marcada participación de enfermeras. Es notable el desarrollo de una construcción teórica rica e inspiradora y de un continuo debate sobre procedimientos metodológicos y marcos interpretativos.
\end{abstract}

El crecimiento de este campo ha sido apoyado por la ramificación de las vías en que las personas buscan diversas formas de mantener su propia salud, de contribuir a su bienestar y de lograr más inclusión social.

Es interesante, a la vez que estimulante, observar un manifiesto interés de parte de las enfermeras en un creciente número de proyectos y ponencias con los que contribuyen a este campo interdisciplinar. Recientemente he tenido ocasión de participar como ponente en el $14^{\circ}$ Congreso bienal de The European Society for Health and Medical Sociology (1) en Hannover, Alemania, al igual que en la $44^{\mathrm{a}}$ conferencia anual del grupo de Sociología Médica de The British Sociological Association (2) en Leicester, Inglaterra. En estos dos encuentros, el debate más prolífico ha surgido de las fuerzas sinérgicas concentradas en asuntos tales como el envejecimiento de las sociedades (3), el seguimiento de marcadores biológicos en su relación con las inequidades sociales (4) englobadas en el plano del capital social (5), y el involucramiento de las enfermeras en asuntos relativos al desarrollo profesional (6) y su vínculo con las categorías sociológicas clásicas, tales como clase social, etnicidad, género, y la conexión de estas categorías con movilidad social y oportunidades de vida. 
Además de la participación en proyectos y ponencias es interesante también su inclusión en comités editoriales de las revistas más influyentes, tales como Social Science \& Medicine, Sociology of Health \& Illnes y Social Theory \& Health. Así también, es de considerable valor el hecho de que las enfermeras forman la principal audiencia de estas revistas interdisciplinares.

Al parecer, en el puente entre la enfermería y las ciencias sociales hay aún un enriquecedor campo por explorar, en miras a un razonamiento expansivo sobre los sistemas de salud, de interés tanto para los profesionales del área como también para los administradores, los diseñadores de políticas y la ciudadanía en general.

\section{REFERENCIAS}

1. Ayala R, Bracke P. Please, mind the gap: social class and ethnicity in health care professions in Latin-America. Resumen presentado en: $14^{\text {th }}$ Conference of the European Society for Health and Medical Sociology. 30 agosto - 1 septiembre 2012; Hannover.

2. Ayala R, Bracke P. Please, mind the gap: social class and ethnicity in healthcare Professions in the Americas. Resumen presentado en: 44th Conference of the British Sociological Association, Medical Sociology Group; 5-7 noviembre 2012; Leicester, UK.

3. Feng Z, Wang WW, Jones K, Li Y. An exploratory multilevel analysis of income, income inequality and self-rated health of the elderly in China. Soc Sci Med. 2012; 75(12): 2481-92.

4. Antunes RJ. The social space of health inequalities in Portugal. Soc Theory Health. 2011; 9(4): 393-409.

5. Palermo TM, Dowd JB. Childhood obesity and human capital accumulation. Soc Sci Med. 2012; 75(11): 1989-98.

6. Timmons S, East L. Uniforms, status and professional boundaries in hospital. Sociol Health Illn. 2011; 33(7): 1035-49. 